\title{
Abbreviation Index
}

\author{
AST Aspartate aminotransferase 147 \\ GPx Glutathion peroxidase 109 \\ GSH glutathion 99 \\ BSO $d l$-buthionine $\{\mathrm{s}, \mathrm{r}\}$-sulfoximine \\ GSH/GSSG Glutathion/glutathion \\ disulfide 153 \\ 99 \\ GSTP Glutathion S-transferase placental \\ CDAA Choline-deficient \\ $L$-amino-acid-defined 153,165 \\ cNOS Constitutive NOS 147 \\ CO Carbon monoxide 114 \\ CSAA Choline-supplemented \\ L-amino-acid-defined 154 \\ CXC 15, 30 \\ CVF Cobra venom factor 86 \\ form 153 \\ $\mathrm{Hb} 114$ \\ $\mathrm{H}_{2} \mathrm{O}_{2}$ Hydrogen peroxide 25, 27, 55 \\ $\mathrm{H}_{2}$ RA 57 \\ $\mathrm{HbO}_{2}$ Free oxyhemoglobin 115 \\ HCC Hepatocellular carcinoma 153, \\ 165 \\ $\mathrm{HCl} 121$ \\ DAF Decay-accelerating factor 82,87 \\ $80 \mathrm{HdG}$ 8-Hydroxydeoxyguanisine 154 , \\ 161,168 \\ $\begin{array}{lll}\text { DFO } & \text { Deferoxamine } 153 \\ \text { DSS } & \text { Dextran sulfate sodium } 34\end{array}$ \\ HO Heme oxigenase 114 \\ HO Hydroxyl radicals 27, 103 \\ EDTA Ethylenediaminetetraacetic acid \\ 106 \\ HOCI Hypochlorous acid 27, 29 \\ HSF Heat shock factor 58 \\ EDTD Ethylenediaminetetraacetic acid \\ 83 \\ HSP Heat shock proteins 58 \\ EMSA Electrophoretic mobility shift \\ assay 36 \\ ET-1 Endothelin-1 42 \\ I/R Ischemia reperfusion $82,94,114$, \\ 120, 147 \\ IBD Inflammatory bowel disease 8 , \\ FA-OOH Fatty acid hydroperoxide $112 \quad 24,34$ \\ $\mathrm{Fe}\left(\mathrm{NO}_{3}\right)_{3}$ iron (III) nitrate nonahydrate IBS Inflammatory bowel syndrome 1, \\ 104


ICAM-1 Intercellular adhesion molecule-1 14, 26

IFN- $\gamma \quad$ Interferon- $\gamma \quad 26$

IL- $1 \beta 46$

IL-8 Interleukin-8 30

iNOS Inducible nitric oxide synthase

99, 120, 136, 147

IPH Ideopathic portal hypertension 115

ISH In situ hybridization 85

JNK jun kinase 143

KC Kupffer cells 18, 116, 121

KCI 104

KOH 105

LDH Lactate dehydrogenase 141

LFA-1 Lymphocyte function-associated antigen-1 (CD11a/CD18) 15, 26, 41

LPO Lipid peroxidation products 16 , 18

LPS Lipopolysaccharides 30, 43, 122

LTB4 Leukotriene B4 25, 26

Mac-1 CD11b/CD18 13, 26, 41, 131

MCP-1 Monocyte chemoattractant protein-1

MDA Malondialdehyde 121, 123, 155

MPT Membrane permeability transition 16

NaCIO 28

NAP Neutrophil-activating protein 29 , 30

NF- $\kappa$ B Nuclear factor- $\kappa$ B $31,120,130$

$\mathrm{NH} 2 \mathrm{Cl}$ Monochloramine 31, 32, 55, 141

NMLA Monomethyl- $L$-arginine 122

NO Nitric oxide $119,136,140,147$

NOS Nitric oxide synthase 28,99 , 116, 119
NSAID(s) Nonsteroidal anti-inflammatory $\operatorname{drug}(\mathrm{s}) \quad 24,32,45$, 52

$\mathrm{O}_{2}^{-} \quad$ Superoxide 28

$\mathrm{O}_{2}^{-} \quad$ Superoxide anion $55,103,141$

$\mathrm{OCI}^{-}$Hypochlorous anion 55

$\mathrm{ONOO}^{-}$Peroxynitrite 28, 137

PAF Platelet activating factor 25

PAGE 84

PBN Phenyl n-tert-buty nitrone 165

PBS Phosphate-buffered saline 83, 84

PC-OOH Phosphatidylcholine

hydroperoxide 104, 106, 111

PECAM-1 Platelet endothelial cell adhesion molecule-1 14

$\mathrm{PGE}_{1} \quad$ Prostaglandin $\mathrm{E}_{1} 44$

$\mathrm{PGE}_{2}$ Prostaglandin $\mathrm{E}_{2}$

PHGPx Phospholipid hydroperoxide glutathione peroxidase 110

PI Peroxidizability index 108

PKA Protein kinase-A 30

PKC Protein kinase-C 30

PLA2 Phospholipase $A_{2} 111$

PMN Polymorphonuclear leukocytes 25

PPI Proton pump inhibitors 57

PTK Protein tyrosine kinase 31

PUFA Polyunsaturated fatty acids 108

ROOH Lipid peroxide 4

ROS Reactive oxygen species 16

SCID Severe combined immunodeficient

8

SIN1 3-Morpholinosydnonimine 141

SMA Superior-mesenteric artery 94

SOD Superoxide dismutase 27, 140 